\title{
Gabapentin regulates dopaminergic neuron firing and theta oscillation in the ventral tegmental area to reverse depression-like behavior in chronic neuropathic pain state
}

This article was published in the following Dove Press journal: Journal of Pain Research

\author{
Bo Fu,' Shao-Nan Wen,' Bin \\ Wang,' Kun Wang, ${ }^{2}$ Ji-Yan \\ Zhang, ${ }^{3}$ Xie-Chuan Weng,' \\ Shao-Jun Liu' \\ 'State Key Laboratory of Proteomics, \\ Department of Neurobiology, Institute \\ of Military Cognitive and Brain Sciences, \\ Academy of Military Medical Sciences, \\ Beijing 100850, China; ${ }^{2}$ Department of \\ Occupational Medicine, Tianjin Institute of \\ Environmental and Occupational Medicine, \\ Tianjin 300050, China; ${ }^{3}$ Department of \\ Molecular Immunology, Institute of Military \\ Cognitive and Brain Sciences, Academy of \\ Military Medical Sciences, Beijing 100850 , \\ China
}

Purpose: Ventral tegmental area (VTA) dopamine system plays an important role in depression and is also involved in pain experience. In this study, we investigated the VTA dopaminergic (DA) neuron firing and local field potential (LFP) in pain-related depression, and we try to explore the underlying relationship between pain and depression.

Materials and methods: We used neuropathic pain model [spare nerve injury (SNI)] to induce pain-related depression. The Dixon up-down method was used to test mechanical hypersensitivity. Behavioral changes like open field test, sucrose preference test, and forced swim test were used to test depression-like behaviors. Gabapentin (GBP) was used to explore the chronic analgesic treatment that could reverse pain-related depression. To investigate the in vivo variations of VTA DA neuron firing and LFP, multichannel acquisition processor system was used. Results: We used SNI to induce depression-like behaviors. Repeated GBP treatment reversed these behaviors after 14 days of injection. An in vivo electrophysiological analysis of the firing characteristics of VTA DA neurons and LFP revealed that SNI increased the firing rate of DA neurons, but not the burst firing activity. Surprisingly, chronic GBP reversed the firing rate of DA neurons and reduced the burst firing activity. Moreover, SNI increased the LFP power in delta and theta oscillation and decreased it in beta oscillation. Repeated administration of GBP significantly suppressed theta oscillation. Above all, chronic GBP altered these characteristics to reverse depression-like behaviors.

Conclusion: The present study confirmed that the tonic firing activity of VTA DA neurons, but not the burst firing activity, was the key factor in peripheral neuropathy-induced depression. Chronic GBP regulated the firing pattern of DA neurons and decreased theta oscillation in VTA to treat pain-related depression. This variation tendency of electrophysiological characteristics of VTA DA neurons and theta oscillation in VTA might represent an attempt to cope with painrelated negative mood disorder.

Keywords: chronic neuropathic pain, depression, dopaminergic neuron, LFP, VTA

\section{Introduction}

Many brain regions, such as the medial prefrontal cortex (mPFC), ventral tegmental area (VTA), and other brain areas, are involved in pain experience, which have important roles in encoding affects and salience. ${ }^{1,2}$ Also, negative emotional experiences, such as depression and anxiety, lead to pain sensation without tissue damage. Neuropathic pain induces multiple transsynaptic changes that extend to the brain. As a result, many patients experience mood disorders such as depression. ${ }^{3}$ The high comorbidity of chronic pain
Correspondence: Shao-Jun Liu;

Xie-Chuan Weng

State Key Laboratory of Proteomics,

Department of Neurobiology, Institute of Military Cognitive and Brain Sciences, Academy of Military Medical Sciences, 27 Taiping Road,

Beijing 100850, China

Email Liusj@nic.bmi.ac.cn;

wengxc2000@sina.com 
syndromes and depression further emphasizes the significance of negative emotions associated with chronic pain. ${ }^{4}$

Pain and depression are two highly extensive disorders. Clinical studies have common views that both coexist and interact. However, the mechanisms underlying pain-depression comorbidity and their dynamic interactions remain largely unknown. Brain imaging studies have shown that the mesolimbic reward circuitry, composed of DA neuron of the VTA and their projections such as mPFC and nucleus accumbens (NAc), is involved in pain sensation and painrelated emotional experiences. ${ }^{5}$ Accumulating evidences indicate that DA neurons modulate acute and chronic pain, as well as the process of pain relief. ${ }^{6,7}$ Recently, metabolic and transcriptional mechanisms in the VTA-NAc dopamine system were shown to regulate depressive behaviors. ${ }^{8-11}$ These evidences indicate that VTA DA system may play an important role in the comorbidity of chronic pain and depression. In electrophysiology, the local field potential (LFP) described neuronal activity in the local tissue, which was composed of low-frequency extracellular voltage fluctuations that reflected synaptic potentials and other slow electrical signals, such as spike after-potentials and voltage-dependent membrane oscillations. ${ }^{3,12}$ Analysis of rhythmic VTA LFP activity could provide a better index to reflect VTA local DA system state. Nevertheless, the mechanisms especially the electrophysiological variation tendency of VTA LFP and DA neurons during the pain-related depression are limited.

Clinically, gabapentin (GBP) is a preferred pain reliever for neuropathic pain. Chronic GBP reduces calcium currents via inhibition of $\alpha_{2} \delta-1$ membrane trafficking, explaining its direct effects on GABA release and misleading nomenclature. ${ }^{13}$ In mesolimbic system, GBP could reduce the dopamine release to prevent cocaine-induced reinstatement and motivation ${ }^{14}$ and impact the functions of midbrain DA system. In this study, a neuropathic pain model [spare nerve injury (SNI)] was used to induce chronic pain stimulus as a long-time nociception to evoke depression-like behaviors. We used GBP as an antalgic to reduce pain, and in particular, we found that repeated GBP could reverse depression-like behaviors by altering the VTA LFP and DA neuron firing patterns. These results suggest that VTA may be one of the key factors in pain-depression comorbidity, provides new avenues to treat this disease, and reveals a potential connection among pain, depression, and reward.

\section{Materials and methods}

\section{Animals}

Male Sprague-Dawley (SD) rats (weighing 200-250 g) were housed in a vivarium under a 12-hour light-dark cycle at a constant temperature $\left(25^{\circ} \mathrm{C}\right)$ and humidity $(50 \%-60 \%)$ with free access to food and water. The rats were acclimatized to the laboratory for 1 week before starting experiments. Moreover, all the experimental procedures were conducted in accordance with the National Institutes of Health Guide for Care and Use of Laboratory Animals and approved by the Institute of Military Cognitive and Brain Sciences Animal Welfare and Ethics Committee.

\section{Experimental design}

As described in Figure 1A, 48 male SD rats were divided into two groups randomly, one group named sham (24 rats), while the other group named SNI (24 rats). Before and after the SNI surgery, in both the groups behavioral test including mechanical allodynia test (six rats one group), open field test (OFT; eight rats one group), sucrose preference test (SPT; eight rats one group), and forced swim test (FST; 10 rats one group) at different time points were conducted. After the last depression-like behaviors test, 12 SNI rats were subjected to repeated GBP injection; the other rats underwent saline treatment as control group. Meanwhile, sham rats were injected with saline. Eighteen rats (six rats one group) were randomly chosen for the depression-like behaviors test to detect the therapeutic effect of chronic GBP. And 27 rats (11 sham rats, $10 \mathrm{SNI}+$ saline rats, and $6 \mathrm{SNI}+\mathrm{GBP}$ rats) were used for electrophysiological recording of VTA DA system.

\section{Drugs and anesthesia}

The rats were anesthetized with Nembutal $(60 \mathrm{mg} / \mathrm{kg}$, intraperitoneally) for SNI surgery and electrophysiological recording. GBP (TCI, G0318, $100 \mathrm{mg} / \mathrm{kg}$, intraperitoneally) was used for pain relief.

\section{Surgery for SNI}

The SNI surgery was conducted as described in detail in a previous study. ${ }^{10}$ Furthermore, rats were anesthetized using Nembutal $(60 \mathrm{mg} / \mathrm{kg})$ intraperitoneally. The skin of the right thigh was incised, and the biceps femoris muscle was dissected to expose the three branches of the sciatic nerve: sural, common peroneal, and tibial nerves. A 5-0 silk suture was used to ligate the common peroneal and tibial nerves. Then, the nerves were cut distal to each knot, and 3-5 mm of the distal ends was removed. In the surgery of sham rats, the aforementioned nerves were exposed but not cut.

\section{Animal behavioral tests Mechanical hypersensitivity test}

Mechanical hypersensitivity was measured using von Frey filaments and the Dixon up-down method. In brief, the rats 


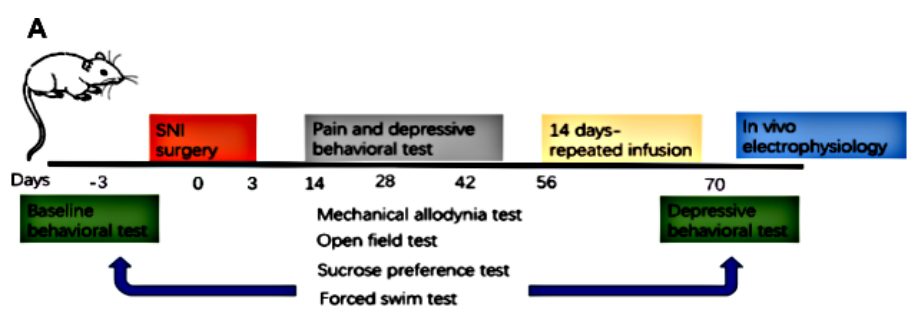

B

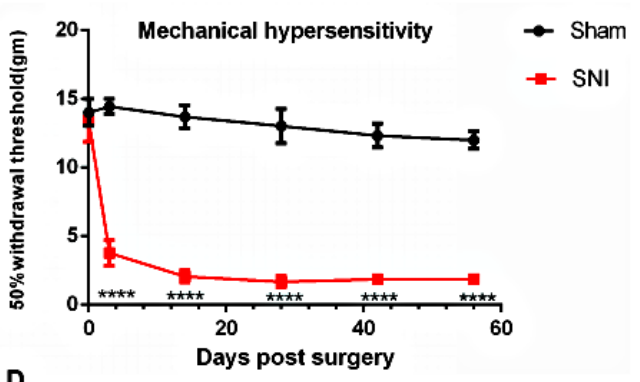

D

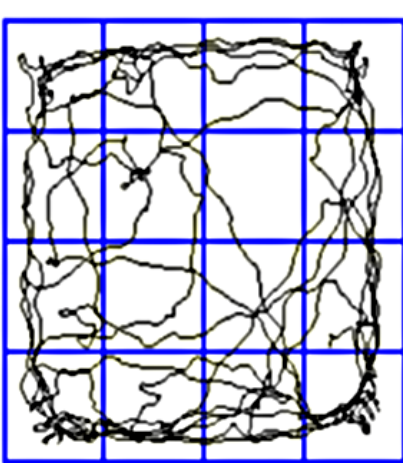

Sham

$\mathbf{F}$

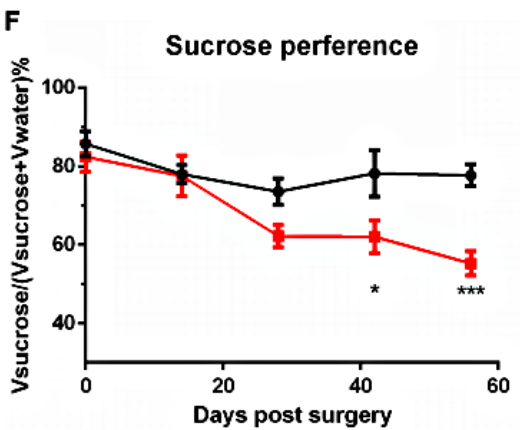

\section{SNI}

$\rightarrow$ SNI
C
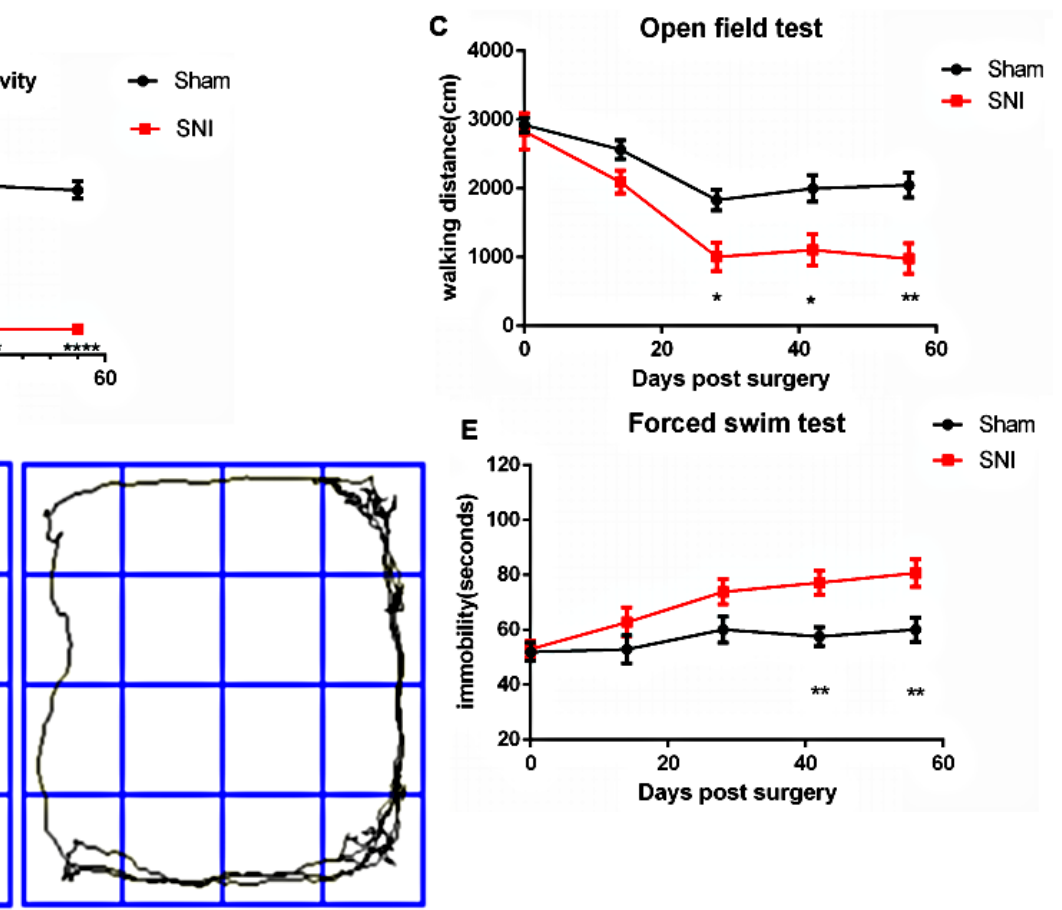

$\rightarrow$ Sham

Figure I SNI caused depression-like behaviors.

Notes: (A) Experimental paradigm. (B) The SNI rats developed mechanical hypersensitivity after surgery compared with the sham rats $(n=6, P<0.0001)$. (C) The SNI rats had significantly decreased mobility with the walking distance in the open field test compared with sham rats on day 28 after SNI surgery, and this lasted till days 42 and 56 compared with the sham rats $(n=8, P=0.0315, P=0.0168, P=0.003)$. (D) Examples of walking trajectory in the open field environment 56 days after $S N I$. The $S N I$ rats developed decreased motivation or behavioral despair, as indicated by prolonged immobility on the forced swim test and preference for sucrose in sucrose preference test on day 42 after SNI surgery, and this lasted till day 56 compared with the sham rats $[(\mathbf{E}) n=10, P=0.0050, P=0.0029 ;(\mathbf{F}) n=8, P=0.0205, P=0.0005]$. Values represented as mean \pm standard

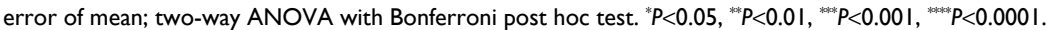

Abbreviation: SNI, spare nerve injury.

were individually placed into Plexiglas chambers over a mesh table and acclimated for 20 minutes before the examination. Beginning with $2.0 \mathrm{~g}$, von Frey filaments in a set with logarithmically incremental stiffness $(0.8,1.0,1.2,1.5,2.0$, $4.0,6.0,8.0,10.0$, and $15.0 \mathrm{~g}$ ) were applied to the lateral one-third of the right paws of rats. ${ }^{15}$ 


\section{OFT}

The OFT apparatus consisted of a noiseproof box $\left(100 \times 100 \times 100 \mathrm{~cm}^{3}\right.$, length $\times$ width $\times$ height). The rats were individually placed in the central area and allowed to explore for 5 minutes. Their activity was video tracked..$^{15}$

\section{SPT}

The rats were acclimated to the test room for $\geq 20$ minutes. Two bottles ( $1 \%$ sucrose solution vs water) were presented to each rat for 30 minutes. The bottles were switched to the opposite side and the test was continued for an additional 30 minutes. At the end of each test, sucrose preference was calculated as the volume of sucrose consumed divided by total liquid consumption for each rat. ${ }^{16}$

\section{FST}

On the first session of the test, each rat was placed in a standard water tank with water at $25^{\circ} \mathrm{C}$ for 15 minutes. After 24 hours, the rat was placed into this chamber under the same conditions for 5 minutes. Immobility was defined as a lack of movement of the hind paws lasting for $>1$ second in the second test. ${ }^{17}$

\section{In vivo electrophysiological recording} Surgical procedure

The surgical and histological procedures were performed as follows: on day 71 following the surgery, the rats were anesthetized with pentobarbital $(60 \mathrm{mg} / \mathrm{kg})$ intraperitoneally and placed in the stereotaxic apparatus (Kopf, Tujunga, CA, USA) with their body temperature maintained at $37^{\circ} \mathrm{C} \pm 1{ }^{\circ} \mathrm{C}$ using a heating pad. Then, the scalp was retracted, and one burr hole was drilled above the area selected for the experiments, according to the Paxinos and Watson (2007) rat brain atlas. In this study, the electrodes were implanted in VTA (anteroposterior, $6.0 \mathrm{~mm}$ from bregma; mediolateral, 0.4-0.6 $\mathrm{mm}$ from midline; dorsoventral, $7.0-8.0 \mathrm{~mm}$ from cortical surface) for all rats. The single unit activity of neurons was extracellularly recorded using a U-probe electrode (16 channels, Plexon Inc., Dallas, TX, USA) when the rats were anesthetized.

\section{Data collection}

The neural activity was amplified, band-pass filtered at $150-8,000 \mathrm{~Hz}$, and continuously sampled at $40 \mathrm{kHz}$ using a multichannel acquisition processor (MAP) system (Plexon Inc.). Spike sorting was performed manually using an Offline Sorter (Plexon Inc.). The data were then transferred to NeuroExplorer (Plexon Inc.) for further analysis. LFP were first band-pass filtered into five bands: delta $(1-4 \mathrm{~Hz})$, theta (4-12 Hz), beta $(12-30 \mathrm{~Hz})$, low gamma $(30-45 \mathrm{~Hz})$, and high gamma $(60-100 \mathrm{~Hz}),{ }^{18}$ and then all data were analyzed using NeuroExplorer (Plexon Inc.). For each rat, the LFP was recorded for at least 15 minutes.

\section{Neuronal classification and analysis}

Immunochemical identification of cell types in extracellular recordings was technically challenging. Differences in the waveform size and shape were used for separating single units. Single putative DA neurons were isolated (band-pass filter $0.1-10.000 \mathrm{~Hz}$ ) and identified according to waveform characterization: 1) action potential with biphasic or triphasic waveform 2.5 milliseconds in duration; 2) 1.1 milliseconds from spike onset to negative trough; and 3) slow spontaneous firing rate $<10$ spikes/s. Bursts were defined as the occurrence of two spikes at interspike interval $<80$ milliseconds and terminated when the interspike interval exceeded 160 milliseconds. ${ }^{19}$

\section{Statistical analysis}

The Student's $t$ test and two-way ANOVA with Bonferroni post hoc test were used for comparing the two treatment groups. One-way and two-way ANOVAs with Tukey's post hoc tests were used for multiple group comparisons. In all figures that contained statistically significant test results, the $P$-value denoted by one asterisk was in the range of $0.05-$ 0.01 , the $P$-value denoted by two asterisks was in the range of $0.01-0.001$, and the $P$-value denoted by three asterisks was $<0.001$. Data were represented as mean \pm standard error of mean. GraphPad PRISM 6.0 was used for statistical analysis.

\section{Results}

\section{Chronic pain led to depression-like behaviors}

This study used the SNI model, a peripheral nerve injury model for chronic neuropathic pain, to examine the regulation of pain-induced depression. Two of three branches of the sciatic nerve were surgically resected, causing permanent nerve injury and neuropathic pain. As the experimental timeline described (Figure 1A), the rats experienced abnormal hypersensitivity to mechanical stimuli 3 days after SNI. Sensory hypersensitivity after SNI was stable, persisting until at least day 56. The mechanical hypersensitivity test was performed 14, 28, 42, and 56 days after surgery [Figure $1 \mathrm{~B} ; F_{\text {group }}(1,60)=347.2, F_{\text {day time }}(5,60)=18.59, F_{\text {interaction }}$ $(5,60)=12.39, P<0.0001]$. In contrast, the control rats that underwent sham operation did not show this sensory hypersensitivity (Figure 1B). 
Next, the depressive symptoms associated with chronic pain were examined. The mobility, anhedonia, and behavioral despair for depression were evaluated using OFT, SPT, and FST, respectively, to demonstrate depression-like behaviors induced by chronic neuropathic pain. The SNI rats showed a significant decrease in walking distance in the OFT compared with the sham rats on day $28(P=0.0315)$, which lasted till days 42 and 56 ( $P=0.0168, P=0.003)$. No difference was observed on day $14\left[P=0.4299\right.$, Figure $1 \mathrm{C} ; F_{\text {group }}$ $(1,70)=30.96, P<0.0001 ; F_{\text {day time }}(4,70)=22.48, P<0.0001$; $\left.F_{\text {interaction }}(4,70)=2.074, P=0.0934\right]$. The walking trajectory of the rats in the open field environment on day 56 after SNI was shorter and less time was spent in the central area compared with those of the sham rats (Figure 1D). In FST, the immobility time significantly increased by SNI on day 42 $(P=0.0050)$ after surgery and lasted till day $56(P=0.0029)$. No difference was observed on days 14 and 28 [ $P=0.4606$, $P=0.0869$, Figure 1E; $F_{\text {group }}(1,90)=21.41, P<0.0001 ; F_{\text {day time }}$ $\left.(4,90)=5.812, P=0.0003 ; F_{\text {interaction }}(4,90)=1.615, P=0.1773\right]$. The SPT was also used to test the depression-like behaviors. The preference for sucrose began to decrease on day 28 after surgery, but with no statistical difference $(P=0.2013)$. A significant decrease was observed on day $42(P=0.0205)$ that lasted till day $56\left[P=0.0005\right.$, Figure $1 \mathrm{~F} ; F_{\text {group }}(1,70)=19.63$, $P<0.0001 ; F_{\text {day time }}(4,70)=7.547, P<0.0001 ; F_{\text {interaction }}$ (4, 70) $=2.778, P=0.0334]$.

Therefore, the experimental data revealed that SNI rats began to demonstrate depressive tendency on day 28 , but they did not display a noticeable difference in the behavioral test. However, on days 42 and 56, a remarkable difference was found in OFT, FST, and SPT, indicating that SNI could induce depression-like behavior. This phenomenon was persistent, stable, and could not be recovered spontaneously.

\section{Repeated injection of GBP reversed depression-like behaviors induced by chronic pain}

This study used GBP, a clinical pain reliever, to study the relationship between chronic pain and depression and examined whether it could treat the depression caused by chronic pain. Therefore, the rats were divided into two groups: one group was treated with GBP and the other with saline injections.

An intraperitoneal injection of GBP $(100 \mathrm{mg} / \mathrm{kg})$ was given to the SNI rats on day 56 after the SNI surgery. GBP suppressed mechanical allodynia after 2 hours (Figure 2A; $t=6.148, P<0.0001)$. Then, the SNI rats were randomly divided into two groups: one group was given a saline injection and the other group was given GBP. According to the experimental design, GBP (100 mg/kg) was infused successfully by intraperitoneal injection once a day. Depressive behavioral tests were conducted on the seventh day of the continuous injection. No difference was found in OFT (Figure 2B, $P=0.6815$ ) and SPT (Figure 2E, $P=0.6410$ ) between the SNI + saline and SNI + GBP group rats. However, in the FST, GBP decreased the immobility time of SNI rats but still had no significant difference compared with SNI + saline group rats (Figure $2 \mathrm{D}, P=0.5357$ ) or sham + saline group rats $(P=0.1711)$. These data indicated that GBP treatment for 7 days could not reverse the depression-like behaviors.

The SNI rats were continuously treated with GBP, and the depressive behavior was evaluated again. Two weeks of successive GBP treatment significantly increased the mobility in SNI rats compared with the SNI + saline group rats $\left[P<0.0002\right.$, Figure $2 \mathrm{~B} ; F_{\text {group }}(2,30)=21.65, P<0.0001$; $F_{\text {day time }}(1,30)=11.69, P=0.0018 ; F_{\text {interaction }}(2,30)=5.407$, $P=0.0099]$; no difference was observed compared with the sham + saline group. The walking trajectory of GBP-treated rats in open field environment on day 14 was longer and more time was spent in the central area compared with the salinetreated groups at the same time (Figure 2C). Chronic GBP also decreased the immobility time during FST in SNI rats compared with the $\mathrm{SNI}+$ saline group rats $(P=0.0476)$; no difference was observed compared with the sham + saline group rats [Figure 2D; $F_{\text {group }}(2,30)=8.630, P=0.0011 ; F_{\text {day time }}$ $\left.(1,30)=1.511, P=0.2286 ; F_{\text {interaction }}(2,30)=1.036, P=0.3672\right]$. Chronic GBP significantly increased the percentage of sucrose preference compared with the SNI + saline group rats $\left[P=0.0133\right.$, Figure $2 \mathrm{E} ; F_{\text {group }}(2,30)=41.53, P<0.0001$; $F_{\text {day time }}(1,30)=1.884, P=0.1800 ; F_{\text {interaction }}(2,30)=3.744$, $P=0.0353$ ], but it was still obviously lower than that in the sham + saline group rats $(P=0.008)$.

\section{Chronic pain stimuli increased the firing rate ofVTA DA neurons, but 14 days of GBP decreased both frequency and burst firing patterns}

This study used the MAP system in vivo extracellular recordings from anesthetized rats to study the firing rates and firing patterns in VTA DA neurons so as to determine the changes in neuronal activity of VTA DA neurons in the chronic neuropathic pain and depression state (Figure 3B). We filtered the DA neurons by its waveform features (Figure 3A). Compared with sham + saline group rats, SNI induced a significant increase in the firing rate of VTA DA neurons $[P=0.0498$, Figure 3C, $F(2,151)=5.087, P=0.0073]$, but not in burst firing patterns $(P=0.2689)$. However, 14 days of 


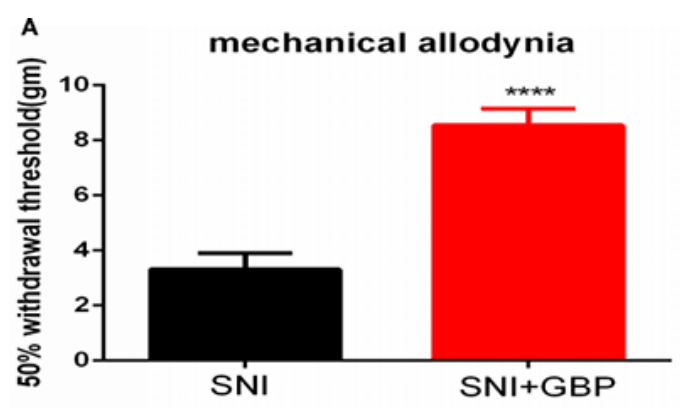

C
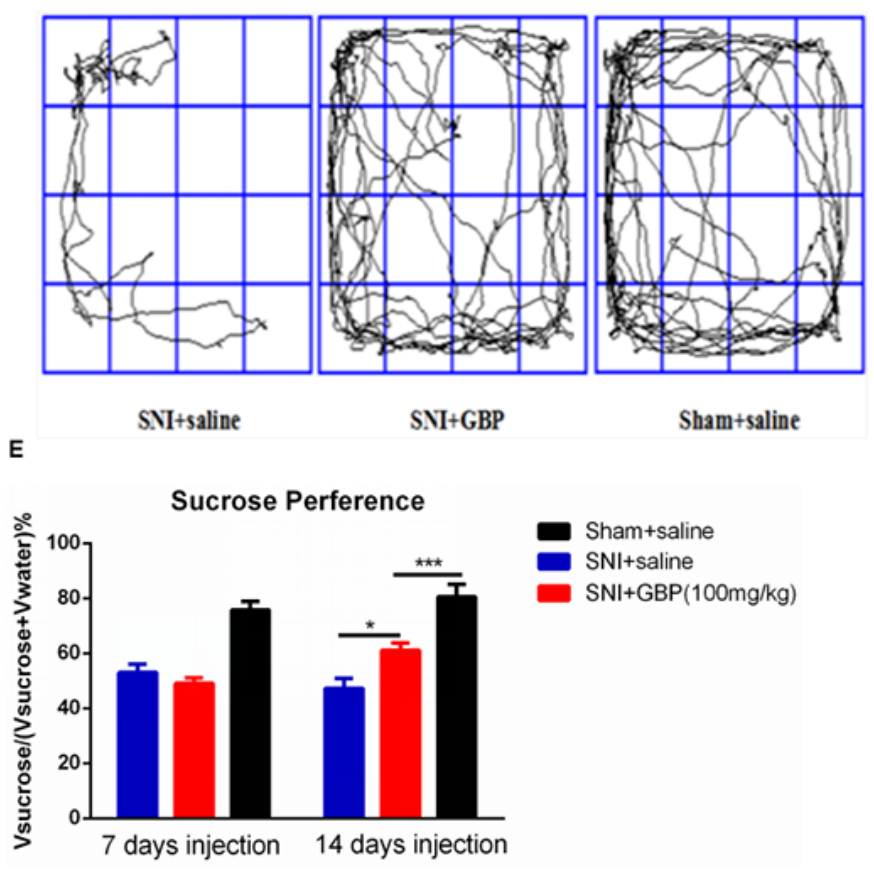

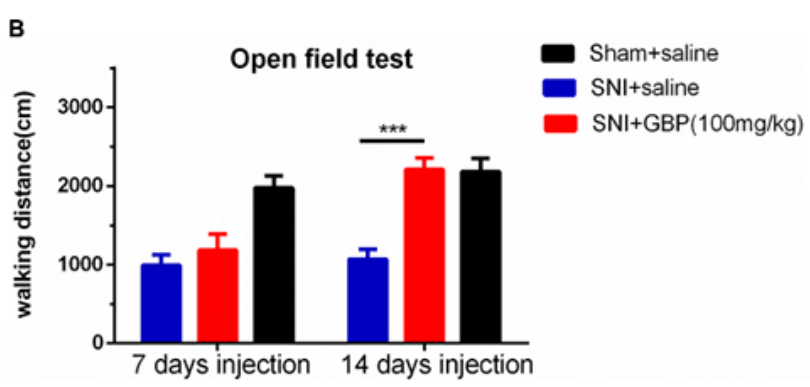

D

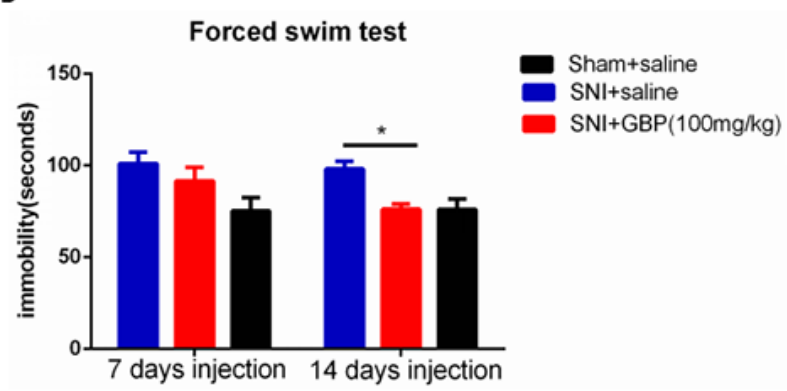

Figure 2 Chronic GBP injection reversed depressive-like behaviors induced by chronic pain.

Notes: (A) Single GBP injection suppressed mechanical allodynia $(n=12$, SNI rats, $P<0.0001)$. (B, D, and E) After 14 days of injection of GBP, SNI rats significantly increased mobility with walking distance in open field environment $[(\mathbf{B}) n=6, P<0.0002]$, decreased behavioral despair, as indicated by prolonged immobility on the FST [(D) $n=6$, $P=0.0476]$, and obviously increased preference for sucrose in the sucrose preference test $[(E) n=6, P=0.0133]$ compared with the SNI rats. (C) The walking trajectory of the rats in open field on day 14 after GBP injection. Values were represented as mean \pm standard error of mean. Student's $t$-test and two-way ANOVA with Tukey's post hoc test were used for comparison between groups. ${ }^{*} P<0.05,{ }^{* * *} P<0.0$ I, ${ }^{* * * *} P<0.00$ I, ${ }^{* * * * * *} P<0.000$ I.

Abbreviations: FST, forced swim test; GBP, gabapentin; SNI, spare nerve injury.

GBP treatment decreased the firing rate of VTA DA neurons $(P=0.0075)$ and \% spikes in bursts $(P=0.0450)$ in SNI, but with no difference compared with the sham group [Figure $3 \mathrm{C}$, $F(2,151)=5.087, P=0.0073$; Figure $3 \mathrm{D}, F(2,112)=3.191$, $P=0.0449]$.

In total, chronic pain stimuli improved the excitability of VTA DA neurons to induce pain-related depression. This might be a clue for pain- and depression-complicated phenomenon in clinical practice. GBP, as a clinical analgesic, was used to treat neuropathic pain. Chronic GBP treatment could decrease the excitability and phasic firing activity of VTA DA neurons to reverse the depression-like behaviors.

\section{Chronic GBP injection decreased VTA theta band oscillation}

The VTA LFP was also examined to test the change in lowfrequency VTA network oscillations $(<250 \mathrm{~Hz})$. The power spectral density percent was used as an indicator of LFP power. The LFP oscillation was divided into five bands: delta oscillation (1-4 Hz), theta oscillation (4-12 Hz), beta oscillation (12-30 Hz), low gamma oscillation $(30-45 \mathrm{~Hz})$, and high gamma oscillation $(60-99 \mathrm{~Hz})$.

In delta oscillation, SNI prominently increased the power spectral density $(P<0.0001)$, and chronic GBP injection decreased this improvement to some degree, but no difference 


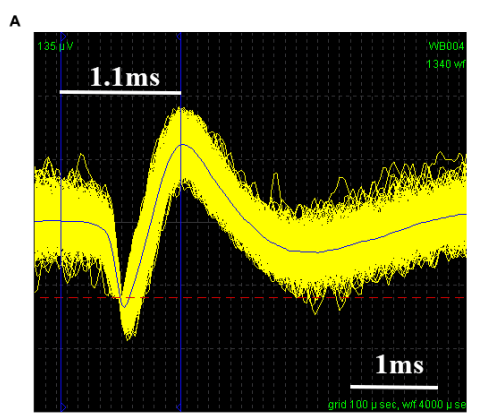

B

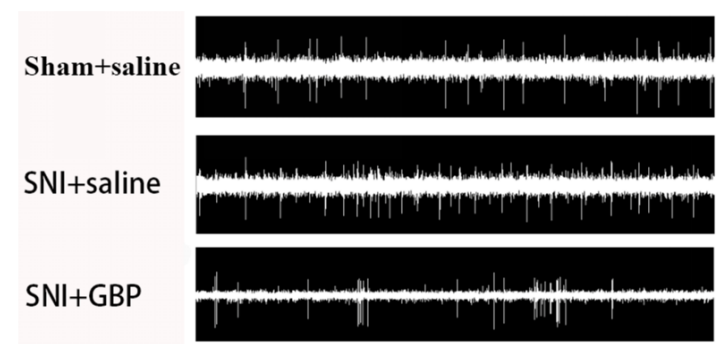

c dopaminergic neuron excitablity

D
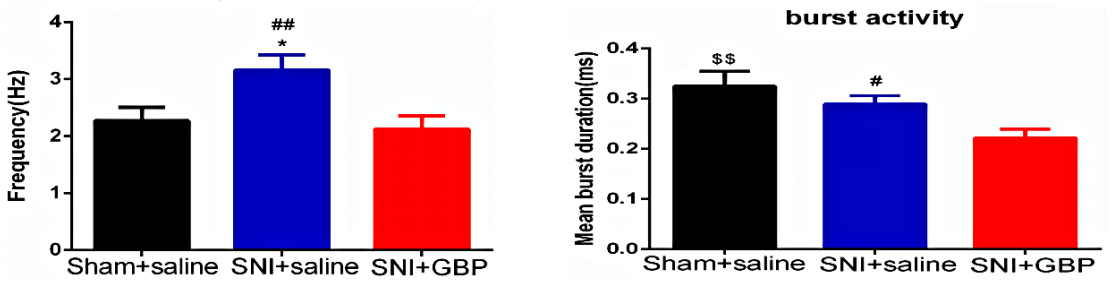

Figure $3 \mathrm{SNI}$ and GBP changed VTA DA neuron firing.

Notes: (A) The waveform pattern of VTA DA neuron. (B) Examples of DA neuron in vivo electrophysiological data in VTA (2 seconds). (C) The in vivo firing rate of DA neurons was significantly increased by SNI compared with sham rats and decreased with 14 days of GBP treatment $[n$ DA neurons $=42$ (sham + saline group rats; $n=I I), 48$ $(\mathrm{SNI}+$ saline group rats; $n=10), 64$ (SNI + GBP group rats; $n=6), P=0.0498, P=0.0075]$; (D) DA neuron burst firing properties, such as \% of spikes in burst, were significantly decreased with chronic GBP treatment compared with SNI rats [n DA neurons=27 (sham + saline group rats; $n=I I), 40$ (SNI + saline group rats; $n=I 0)$, 48 ( $S N I+G B P$ group rats; $n=6), P=0.0073$ ]. Values were reported as mean \pm standard error of mean. One-way ANOVA with Tukey's post hoc test was used for comparison between groups;

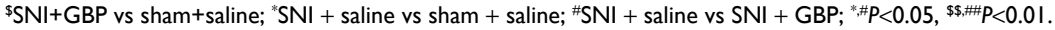

Abbreviations: DA, dopaminergic; GBP, gabapentin; SNI, spare nerve injury; VTA, ventral tegmental area.

was found $(P=0.1123)$. Both these groups had a significant difference compared with the sham rats $[P=0.0053$; Figure $4 \mathrm{~B}, F(2,232)=22.45, P<0.0001]$. In theta oscillation, the LFP power was significantly increased by SNI compared with the sham rats $(P<0.0001)$, and repeated GBP treatment could remarkably reverse this change $[P=0.0002$; Figure $4 \mathrm{D}$, $F(2,232)=16.08, P<0.0001]$. The average LFP power spectra of $1-12 \mathrm{~Hz}$ network oscillation in linear graphs are shown in Figure $4 \mathrm{~A}$ and $\mathrm{C}$.

In the beta band, the LFP power was reduced by chronic pain $(P=0.002)$ and GBP treatment $(P<0.0001)$ compared with the sham rats, and no difference was found between both groups $[P=0.068$; Figure 4F, $F(2,232)=12.95, P<0.0001]$. The average LFP power spectra of 12-30 Hz network oscillation in linear graphs are shown in Figure 4E. The gamma band was divided into low gamma band (30-45 Hz) and high gamma band (60-99 Hz). SNI reduced the power spectral density in both low gamma $(P=0.5060)$ and high gamma band oscillations ( $P=0.4765)$, but no difference was found between the SNI and sham groups. Furthermore, 14 days of GBP injection also decreased the LFP power and had no difference compared with SNI rats in low gamma $(P=0.0662)$ and high gamma band oscillations $(P=0.7637)$. However, compared with the sham group, GBP-treated rats had a significant difference in low gamma band oscillation $[P=0.0083$; Figure 4H, $F(2,223)=4.542, P=0.0117]$ and no difference in high gamma band oscillation $[P=0.2620$; Figure $4 \mathrm{~J}, F(2,225)=1.370 ; P=0.2562]$. The average LFP power spectra of low gamma $(30-45 \mathrm{~Hz})$ and high gamma (60-99 Hz) network oscillations in linear graphs are shown in Figure $4 \mathrm{G}$ and $\mathrm{I}$.

\section{Discussion}

Neuropathic pain is commonly associated with affective disorders such as anxiety and depression. ${ }^{20-22}$ Previous studies have demonstrated measures of depression-like behaviors in rodent models of neuropathic pain, ${ }^{15,23}$ yet, few have determined if the hypersensitivity indicative of a pain state is actually correlated with the depressive behavior observed. The present study used a rodent neuropathic pain model to examine whether the pain caused depression-like behaviors. The study had three key findings. First, SNI caused depression-like behaviors and this behavioral change could not be reversed without drug or other treatments. Second, repeated administration of GBP for at least 14 days reversed depression-like behaviors induced by chronic pain. Third, SNI could significantly increase VTA DA neuron firing rate and low-frequency oscillation, which were associated with 

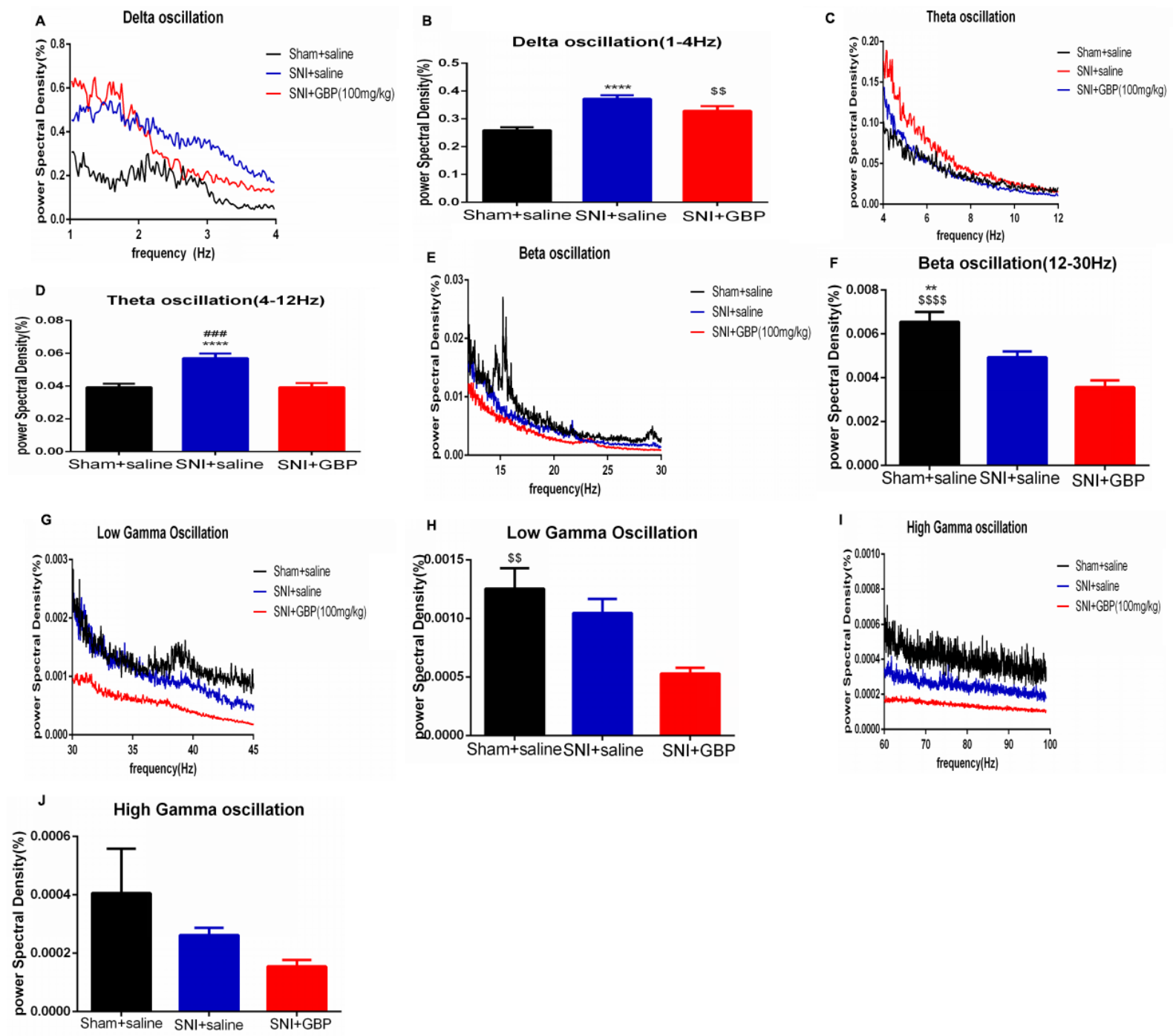

Figure 4 Chronic GBP injection decreased VTA theta band oscillation.

Notes: (A, C, E, G, and I) Linear graphs show average LFP power spectra (I-12 Hz; A), beta oscillation (I2-30 Hz; E), low gamma (30-45 Hz; G), and high gamma (60-99 $\mathrm{Hz}$; I) oscillations for sham + saline group rats, SNI + saline group rats, and SNI + GBP group rats. (B, D, F, H, and J) Bar graphs represent the mean total LFP power in sham + saline, SNI + saline, and SNI + GBP group rats within five frequency band ranges: delta, theta, beta, low gamma, and high gamma oscillations. In the delta band, the LFP power was significantly increased by chronic pain, and repeated GBP reversed the change, but no difference was found compared with the SNI group. Both groups had significant difference compared with the sham group [ $B ; n=I I$ (sham + saline), $n=10$ (SNI + saline), $n=6$ (SNI + GBP); $P<0.000 I, P=0.0053]$. In theta band oscillation, SNI increased the LFP power and chronic GBP reversed the change [D; $n=1$ I (sham + saline), $n=10$ (SNI + saline), $n=6$ (SNI + GBP); $P<0.000 I, P=0.0002]$; SNI and chronic GBP significantly decreased LFP power in beta oscillation compared with the sham group [F; $n=I I$ (sham + saline), $n=10$ (SNI + saline), $n=6$ (SNI + GBP); $P=0.002, P<0.000$ I]. Chronic GBP decreased the power of low gamma oscillations compared with the sham rats $[\mathbf{H} ; \mathrm{n}=\mathrm{II}$ (sham + saline), $\mathrm{n}=10$ (SNI + saline), $\mathrm{n}=6$ (SNI + GBP); $P=0.0083]$. No difference was observed in high gamma oscillation between these three groups [J; $n=I I$ (sham + saline), $n=10$ (SNI + saline), $n=6$ (SNI + GBP); P>0.05]. Values were reported as mean \pm standard error of mean. One-way ANOVA with Tukey's post hoc test was used for comparison between groups. "SNI + saline vs sham + saline; \#SNI + saline vs SNI + GBP; $\$ S N I+G B P$ vs sham + saline; ${ }^{* * \$ \$} \$ \$<0.01,{ }^{\infty}+\cdots<0.001 ;$

Abbreviations: GBP, gabapentin; LFP, local field potential; SNI, spare nerve injury; VTA, ventral tegmental area.

depression-like behaviors. Meanwhile, chronic GBP injection reversed this phenomenon by suppressing DA neuron firing rate and VTA theta band oscillation.

Mechanical hypersensitivity is an indication of chronic neuropathic pain, and behavioral despair (FST), anhedonia (SPT), and mobility (OFT) serve as indices for mood disorder. ${ }^{24,25}$ In the present study, SNI-induced mechanical allodynia and depressive-like behaviors were confirmed by increased withdrawal threshold and immobility time as well as reduced sucrose preference, which suggested that SNI induced the comorbidity of chronic pain and depression. ${ }^{15,16}$ Previous studies indicate that SNI surgery induced depression-like behaviors in the early days $\left(7\right.$ day $^{16}$ or 14 days $^{15,26}$ after surgery). However, the present study found that depression-like behaviors, such as walking distances in OFT, immobility time in FST, and sucrose preference in SPT, began to decrease on day 28 after surgery, but no statistical difference was found. However, significant difference was 
observed on day 42 after surgery and persisted up to day 56. Moreover, only the SNI rats developed depression-like behaviors.

How pain caused depression was not well understood. Neuropathy from SNI creates chronic nociceptive stimulus, causing depression. ${ }^{5}$ Also, pain stimulus was shown to impact the synaptic connectivity in $\mathrm{mPFC},{ }^{6,27,28}$ accumbens nucleui, ${ }^{7,15}$ and dopamine signal pathway from the VTA. ${ }^{29}$ To date, accumulating evidences suggest that midbrain DA neurons modulate acute and chronic pain. Clinical pain reliever can also cure spontaneous pain, anxiety or depression-like behaviors. ${ }^{30-32}$ The present study used a single injection of GBP to inhibit neuropathic pain, followed by chronic injection for 14 days. It was found that SNI-induced depression-like behaviors were reversed. These findings indicated that pain-induced depression could be treated with chronic analgesic injection.

SNI was reported to increase burst activity, but not tonic firing in VTA DA neurons in the early days after surgery (14 days). ${ }^{33}$ However, the present study found that SNI increased the firing rate of DA neurons, but not the burst firing activity compared with sham group when rats exhibited depression-like behaviors by a long-time nociceptive stimulus. Dopamine is known to be important in reward and motivation. Anhedonia and despair are common core symptoms of depression. ${ }^{34}$ Dysfunction of midbrain DA system was a result of anhedonia and depression. ${ }^{35}$ Different kinds of stress, such as chronic mild unpredictable stress (CMS) and chronic social defeat stress (CSDS), established depression, inducing opposing changes in VTA DA neuron activity: CMS reduced the firing rate in VTA DA neurons, whereas susceptibility to CSDS was accompanied by an increase in the firing rate. Moreover, chronic GBP decreased the firing rate of DA cells and burst firing activity at the same time to reverse the depression-like behaviors. Our data provide a clue for modulating firing of VTA DA neurons that could prevent the development of pain-related depression-like behaviors.

GBP interacts with a subunit of voltage-sensitive calcium $\left(\mathrm{Ca}^{2+}\right)$ channels ${ }^{36}$ and modulates the function of peripheral and central pain pathways by influencing fast synaptic transmission and neuronal excitability. The VTA LFP was measured to explore the low-frequency extracellular voltage fluctuations so as to further explore the relationship among chronic pain stimuli, chronic GBP treatment, and VTA DA cells, which reflected synaptic potentials to see how chronic pain and drug affected VTA cells to induce and reverse the depression. Recent studies showed that the delta band (1-4 $\mathrm{Hz}$ ) represented slow-wave sleep. The amplitude of theta oscillations (4-12 Hz) was related to spatial memory in animal models. ${ }^{37,38}$ Beta and gamma oscillations (12-100 $\mathrm{Hz}$ ) were involved in episodic memory in humans. ${ }^{39} \mathrm{~A}$ recent study indicated that the beta oscillation was more relevant for long-range synchronization involving long transmission delays, ${ }^{40}$ whereas the gamma oscillation was more evident in the communication of close-by areas. ${ }^{41}$ Cocaine was reported to significantly increase VTA theta band oscillations in freemoving rats, ${ }^{42}$ indicating delta and theta oscillations in VTA corresponded to motivation. The present study found that SNI increased the LFP power in delta, theta, and beta oscillations, but GBP significantly decreased the theta oscillation, indicating that chronic GBP treatment reduced theta band oscillation to reverse the depression-like behaviors at the level of LFP. The present findings provided a new direction to understand the relationship between pain and depression at the level of LFP.

\section{Conclusion}

The present study confirmed that the tonic firing activity of VTA DA neurons, but not the burst firing activity, was the key factor in peripheral neuropathy-induced depression. Chronic GBP regulated the firing pattern of DA neurons and decreased theta oscillation in VTA to treat pain-related depression. The functional consequences of VTA DA and depression induced by chronic pain remain controversial. Nonetheless, the variation tendency of VTA DA neurons plasticity and theta oscillation represented an attempt to cope with pain-related negative mood experience positively. It provided a new direction to understand the mechanism of pain-induced depression.

\section{Disclosure}

The authors report no conflicts of interest in this work.

\section{References}

1. Elman I, Borsook D, Volkow ND. Pain and suicidality: insights from reward and addiction neuroscience. Prog Neurobiol. 2013;109:1-27.

2. Mitzdorf U. Properties of the evoked potential generators: current source-density analysis of visually evoked potentials in the cat cortex. Int J Neurosci. 1987;33(1-2):33-59.

3. Metz AE, Yau HJ, Centeno MV, Apkarian AV, Martina M, Merzenich MM. Morphological and functional reorganization of rat medial prefrontal cortex in neuropathic pain. Proc Natl Acad Sci U SA. 2009;106(7): 2423-2428.

4. Lammel S, Ion DI, Roeper J, Malenka RC. Projection-specific modulation of dopamine neuron synapses by aversive and rewarding stimuli. Neuron. 2011;70(5):855-862.

5. Zhang H, Qian YL, Li C, et al. Brain-derived neurotrophic factor in the mesolimbic reward circuitry mediates nociception in chronic neuropathic pain. Biol Psychiatry. 2017;82(8):608-618.

6. Wood PB. Mesolimbic dopaminergic mechanisms and pain control. Pain. 2006;120(3):230-234.

7. Schwartz N, Temkin P, Jurado S, et al. Chronic pain. Decreased motivation during chronic pain requires long-term depression in the nucleus accumbens. Science. 2014;345(6196):535-542. 
8. Chang $\mathrm{CH}$, Grace AA. Amygdala-ventral pallidum pathway decreases dopamine activity after chronic mild stress in rats. Biol Psychiatry. 2014;76(3):223-230.

9. Russo SJ, Nestler EJ. The brain reward circuitry in mood disorders. Nat Rev Neurosci. 2013;14(9):609-625.

10. Cao JL, Covington HE, Friedman AK, et al. Mesolimbic dopamine neurons in the brain reward circuit mediate susceptibility to social defeat and antidepressant action. J Neurosci. 2010;30(49):16453-16458.

11. Tye KM, Mirzabekov JJ, Warden MR, et al. Dopamine neurons modulate neural encoding and expression of depression-related behaviour. Nature. 2013;493(7433):537-541

12. Kodama D, Ono H, Tanabe M. Altered hippocampal long-term potentiation after peripheral nerve injury in mice. Eur $J$ Pharmacol. 2007;574(2-3):127-132.

13. Taylor CP, Gee NS, Su TZ, et al. A summary of mechanistic hypotheses of gabapentin pharmacology. Epilepsy Res. 1998;29(3):233-249.

14. Spencer S, Brown RM, Quintero GC, et al. $\alpha 2 \delta$-1 signaling in nucleus accumbens is necessary for cocaine-induced relapse. J Neurosci. 2014;34(25):8605-8611.

15. Lee M, Manders TR, Eberle SE, et al. Activation of corticostriatal circuitry relieves chronic neuropathic pain. $J$ Neurosci. 2015;35(13):5247-5259.

16. Wang J, Goffer Y, Xu D, et al. A single subanesthetic dose of ketamine relieves depression-like behaviors induced by neuropathic pain in rats. Anesthesiology. 2011;115(4):812-821.

17. Chaudhury D, Walsh JJ, Friedman AK, et al. Rapid regulation of depression-related behaviours by control of midbrain dopamine neurons. Nature. 2013;493(7433):532-536.

18. Gazit T, Friedman A, Lax E, et al. Programmed deep brain stimulation synchronizes VTA gamma band field potential and alleviates depressivelike behavior in rats. Neuropharmacology. 2015;91:135-141.

19. Juarez B, Morel C, Sm K, et al. Midbrain circuit regulation of individual alcohol drinking behaviors in mice. Nat Commun. 2017;8(1):2220.

20. Daniel HC, Narewska J, Serpell M, Hoggart B, Johnson R, Rice ASC. Comparison of psychological and physical function in neuropathic pain and nociceptive pain: Implications for cognitive behavioral pain management programs. Eur J Pain. 2008;12(6):731-741.

21. Smith BH, Torrance N, Bennett MI, Lee AJ. Health and quality of life associated with chronic pain of predominantly neuropathic origin in the community. Clin J Pain. 2007;23(2):143-149.

22. Miller LR, Cano A. Comorbid chronic pain and depression: who is at risk? J Pain. 2009;10(6):619-627.

23. Hu Y, Yang J, Hu Y, Wang Y, Li W. Amitriptyline rather than lornoxicam ameliorates neuropathic pain-induced deficits in abilities of spatial learning and memory. Eur J Anaesthesiol. 2010;27(2): $162-168$.

24. Edita N, Xie JY, Alec O, et al. Pain relief produces negative reinforcement through activation of mesolimbic reward-valuation circuitry. Proc Natl Acad Sci U S A. 2012;109:20709.

25. Saadé NE, Atweh SF, Bahuth NB, Jabbur SJ. Augmentation of nociceptive reflexes and chronic deafferentation pain by chemical lesions of either dopaminergic terminals or midbrain dopaminergic neurons. Brain Res. 1997;751(1):1-12.
26. Ning $X$, Tang $X H$, Wei $P$, et al. Spared nerve injury increases the expression of microglia M1 markers in the prefrontal cortex of rats and provokes depression-like behaviors. Front Neurosci. 2017;11:209.

27. Geha PY, Baliki MN, Harden RN, Bauer WR, Parrish TB, Apkarian AV. The brain in chronic CRPS pain: abnormal gray-white matter interactions in emotional and autonomic regions. Neuron. 2008;60(4):570-581.

28. Shen H-Wei, Gipson CD, Huits M, Kalivas PW, Shen H. Prelimbic cortex and ventral tegmental area modulate synaptic plasticity in nucleus accumbens during cocaine-reinstated drug seeking. Neuropsychopharmacology. 2014;39(5):1169-1177.

29. Li X, Huang M, Yang L, et al. Overexpression of thioredoxin-1 blocks morphine-induced conditioned place preference through regulating the interaction of $\gamma$-aminobutyric acid and dopamine systems. Front Neurol. 2018;9:309.

30. Zhang M-M, Liu S-B, Chen T, et al. Effects of NB001 and gabapentin on irritable bowel syndrome-induced behavioral anxiety and spontaneous pain. Mol Brain. 2014;7(1):47.

31. Valente MM, Bortolotto V, Cuccurazzu B, et al. 2 ligands act as positive modulators of adult hippocampal neurogenesis and prevent depressionlike behavior induced by chronic restraint stress. Mol Pharmacol. 2012;82(2):271-280.

32. Leknes S, Tracey I. A common neurobiology for pain and pleasure. Nat Rev Neurosci. 2008;9(4):314-320.

33. Friedman AK, Walsh JJ, Juarez B, et al. Enhancing depression mechanisms in midbrain dopamine neurons achieves homeostatic resilience. Science. 2014;344(6181):313-319.

34. Krishnan V, Han M-H, Graham DL, et al. Molecular adaptations underlying susceptibility and resistance to social defeat in brain reward regions. Cell. 2007;131(2):391-404.

35. Sagheddu C, Aroni S, de Felice M, et al. Enhanced serotonin and mesolimbic dopamine transmissions in a rat model of neuropathic pain. Neuropharmacology. 2015;97:383-393.

36. Taylor CP. Mechanisms of action of gabapentin. Rev Neurol. 1997; 153(Suppl 1):S39.

37. O'Neill P-K, Gordon JA, Sigurdsson T. Theta oscillations in the medial prefrontal cortex are modulated by spatial working memory and synchronize with the hippocampus through its ventral subregion. $J$ Neurosci. 2013;33(35):14211-14224.

38. Kaplan R, Bush D, Bonnefond M, et al. Medial prefrontal theta phase coupling during spatial memory retrieval. Hippocampus. 2014;24(6):656-665.

39. Nyhus E, Curran T. Functional role of gamma and theta oscillations in episodic memory. Neurosci Biobehav Rev. 2010;34(7):1023-1035.

40. Kopell N, Ermentrout GB, Whittington MA, Traub RD. Gamma rhythms and beta rhythms have different synchronization properties. Proc Natl Acad Sci U S A. 1867;2000:97.

41. Gregoriou GG, Gotts SJ, Zhou H, Desimone R. High-frequency, longrange coupling between prefrontal and visual cortex during attention. Science. 2009;324(5931):1207-1210.

42. Koulchitsky S, Delairesse C, Beeken T, et al. Activation of D2 autoreceptors alters cocaine-induced locomotion and slows down local field oscillations in the rat ventral tegmental area. Neuropharmacology. 2016;108:120-127.
Journal of Pain Research

\section{Publish your work in this journal}

The Journal of Pain Research is an international, peer reviewed, open access, online journal that welcomes laboratory and clinical findings in the fields of pain research and the prevention and management of pain. Original research, reviews, symposium reports, hypothesis formation and commentaries are all considered for publication.

\section{Dovepress}

The manuscript management system is completely online and includes a very quick and fair peer-review system, which is all easy to use. Visit http://www.dovepress.com/testimonials.php to read real quotes from published authors. 